\title{
THEORETICAL HIGH ENERGY PHYSICS RESEARCH AT THE UNIVERSTTY OF CHICAGO
}

Progress Report

for period October 1, 1991 - April 30, 1992

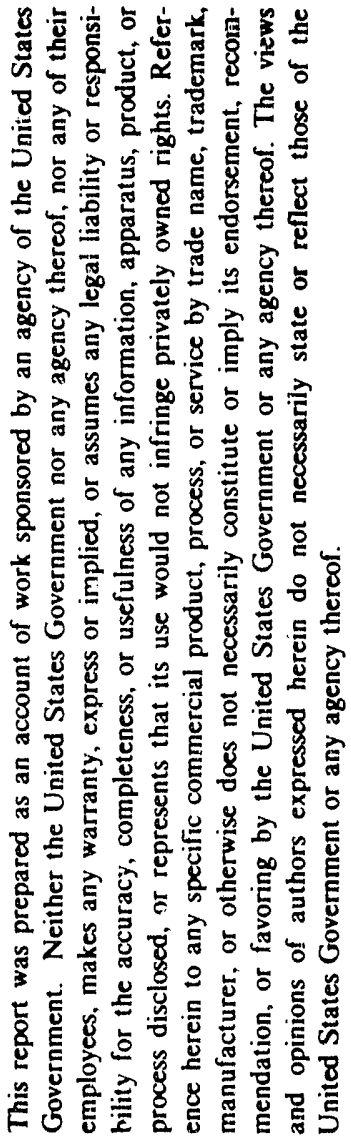

\author{
JONATHAN L. ROSNER \\ EMIL J. MARTINEC \\ ROBERT G. SACHS
}

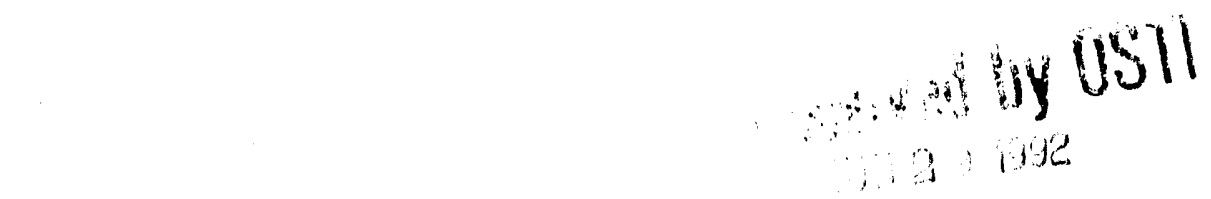

The University of Chicago

5801 South Ellis Avenue

Chicago, Illinois 60637

April, 1992

Prepared for

THE U.S. DEPARTMENT OF ENERGY AGREEMENT NO. DE-FG02-90ER-40560

MMSTER

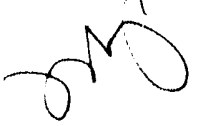




\section{TECHNICAL PROGRESS REPORT}

This report was prepared as an account of work sponsored by the United States Government. Neither the United States nor the United States Department of Energy, nor any of their employees nor any of their contractors, subcontractors, or their employees makes any warranty, express or implied, or assumes any legal liability or responsibility for the accuracy, completeness, or usefulness of any information, apparatus, product or process disclosed or represents that its use would not infringe privately owned rights.

DOE DE FG02 90-ER 40560

TECHNICAL PROGRESS REPORT

Theoretical High Energy Physics Research

at the University of Chicago

\section{INTRODUCTION}

The present contract supported work by Jonathan E. Rosner (principal investigator), Ernil J. Martinec (co-investigator) Robert (G. Sachs (coinvestigator), Research Associates Peter Bowcock and Glenn Boyd, and graduate students in elementary particle physics at the University of Chicago.

The present report deals with work pe:formed during the last three months of Calendar Year 1991 and the first four months of Calendar Year 1992. For earlier work, see previous Technical Progress Reports.

Dr. Chris Hill of Fermilab is teaching a course on electroweak physics at the University of Chicago during three months in the spring of 1992. He is interacting fruitfully with members of the theoretical and experimental high energy physics group. 


\section{RESEARCH ACTIVITIES}

\section{A. J. ROSNER, COLLABORATORS, AND STUDENTS}

J. Rosner participated in a workshop on $b$ physics at the University of Edinburgh in December, 1991. He delivered an overview of electroweak physics at a HEPAP subpanel review of Fermilab in February, 1992.

\section{CP violation and Cabibbo-Kobayashi-Maskawa matrix.}

An update of a joint work with C. S. Kim and C.-P. Yuan ${ }^{1}$ was performed in collaboration with Geoffrey Harris, now at Syracuse University. Recent lattice gauge theory calculations of the $B$ meson decay constani $f_{B}$ indicate a larger value $\left(f_{B} \simeq 1.7 f_{x}\right)$ than potential model calculations $\left(f_{B} \simeq f_{\pi}\right)$. The data on CP violation in the kaon system, charmless $b$ decays, and $B-\bar{B}$ mxing permit either value at present. Experimenis which would shed further light on the value of $f_{B}$ and on CKM matrix elements were studied. This work has now been published. ${ }^{2}$

A review of the CKM matrix was completed and is to be published in a jook on $B$ decays. ${ }^{3}$

Further investigations regarding possible quark mass matrices leading to the observed form of the CKM matrix were performed in collaboration with Mihir Worah, a graduate student at Chicago. Hamiltonians leading to a hierarchical structure of the quark mass matrices were constructed. These Hamiltonians are based on models of quarks as composites of subunits which interact via spin-spin forces. The work has been submitted for publication. ${ }^{4}$

The review of Ref. 3 was condensed, adapted for the special case of $b$ physics, and presented at a workshop in Edinburgh in December, 1991. ${ }^{5}$ At that workshop, in collaboration with Paul Harrison, it was found that ratios of observables for $B$ decays carried useful information about the structure of 
the CKM matrix. This work has been submitted for publication. ${ }^{6}$

2. Radiative corrections and electroweak observables

A general description of the impact of electroweak measurements makes use of variables $S$ and $T$ (defined in Ref. 7) which parametrize "oblique" radiative corrections. In this context it was pointed out ${ }^{8}$ that atomic parity violation experiments provide useful information on $S$. Within the context of the standard electroweak theory with a single Higgs doublet, however, a more physical set of variables consists of the masses of the top quark $\left(m_{t}\right)$ and $W$ boson $\left(M_{W}\right)$. A brief review of the impact of various measurements for specification of regions in the $\left(m_{t}, M_{W}\right)$ plane was prepared for the new section ("Colloquia") of Reviews of Modern Physics." This review, incorporating later LEP data, represents an updating of a previous one. ${ }^{10}$

\section{Heavy quark symmetry}

J. Amundson, a graduate student at Chicago, is currently studying patterns of deviations from heavy quark symmetry ${ }^{11}$ in charmed particle decays. These deviations, expected to be appreciable, can provide warnings of deviations in $b$ decays, thereby helping to specify experimental errors in the determination of the CKM matrix elements $\left|V_{c b}\right|$ and $\left|V_{u b}\right|$. At the moment, attention is focused on a particular set of $1 / m$ corrections which arise from spin-spin interactions in the final states of such processes as $D \rightarrow K l \nu$ and $D \rightarrow K^{*} l \nu$. This work is continuing.

\section{Heavy meson spectroscopy}

In collaboration with Eric Rynes, as a follow-up on his Senior Thesis ${ }^{12}$ (for which J. Rosner was the advisor), a study is being performed of the optimum power-law potential $V=\lambda r^{\alpha}$ which can describe both $c \bar{c}$ and $b \bar{b}$ systems. This work represents an updating of earlier investigations ${ }^{13,14}$ in 
the light of improved information on $P$-wave $b \bar{b}$ levels.

Work by Aaron Grant, a new graduate student is being supervised. Mr. Grant has studied the relative strengths of electric dipole transitions with $\Delta L=1$ between states with various numbers of nodes $n_{r}$ in the radial wave function. He finds for power-law potentials $V=\lambda r^{\alpha} / \alpha$ that the ratio $\left\langle l, n_{r}=0|r| l-1, n_{r}=2\right\rangle /\left\langle l, n_{r}=0|r| l+1, n_{r}=0\right\rangle$ behaves as $1 / l$ for large $l$. The coefficient of $1 / l$ depends on $\alpha$ and reduces to 1 for the Coulomb case $\alpha=-1$. This work is continuing.

5. Hadronic string theory

The work by P. Freund and J. Rosner mentioned in the previous Technical Progress Report has been published. ${ }^{15}$

6. Composite models of quarks and leptons

Work with Davison Soper ${ }^{16}$ and with Gerard Jungman, ${ }^{17}$ mentioned in the previous Technical Progress Report, has been published. The study of a composite model of quarks and its implications for the CKM matrix were mentioned above. ${ }^{4}$

\section{Pedagogical efforts}

In a graduate course taught last year on Electrodynamics, students were encouraged to do experiments for their final projects. A number of good papers were submitted, ${ }^{18}$ including the study of electromagnetic resonances in a coffee can.

In following up topics associated with the above course (Physics 324), it was noted that the Smith Chart, a graph used for antenna impedance matching, represents a simple conformal transformation of complex impedance, normalized by that of the transmission line. It was realized that such a transformation has its analogy in quantum mechanics and may be used to 
motivate phase factors in the WKB approximation. An article to this effect was submitted to The American Journal of Physics. ${ }^{19}$

1. C. S. Kim, Jonathan L. Rosner and C.-P. Yuan, Phys. Rev. D 42, 96 (1990); 45, 389(E) (1992).

2. Geoffrey R. Harris and Jonathan L. Rosner, Phys. Rev. D 45, 946 (1992).

3. Jonathan L. Rosner, Enrico Fermi Institute report EFI 91-49-Rev., September, 1991, to appear as a chapter of the book $B$ Decays, edited by Sheldon L. Stone (World Scientific, Singapore, 1992).

4. Jonathan L. Rosner and Mihir Worah, Enrico Fermi Institute report EFI 92-12, March, 1992, submitted to Phys. Rev. D.

5. Jonathan L. Rosner, Enrico Fermi Institute report EFI 92-02, January, 1992, to be published in J. Phys. G.

6. Paul F. Harrison and Jonathan L. Rosner, Enrico Fermi Institute report EFI 92-05, February, 1992, to be published in J. Phys. G.

7. Michael E. Peskin and Tatsu Takeuchi, Phys. Rev. Lett. 65, 964 (1990); Phys. Rev. D 45, to be published.

8. William J. Marciano and Jonathan L. Rosner, Phys. Rev. Lett. 65, 2963 (1990); 68, 898(E) (1992).

9. Jonathan L. Rosner, Enrico Fermi Institute report EFI 92-19, April, 1992, to be submitted to Rev. Mod. Phys. (Colloquia). 
10. Jonathan L. Rosner, in Intersections between Particle and Nuclear Physics, Proceedings of $4^{\text {th }}$ Conference, Tucson, AZ, May 24-29, 1991, edited by W. T. H. van Oers (AIP, New York, 1992), p. 156.

11. M. B. Voloshin and M. A. Shifman, Yad. Fiz. 45, 463 (1987) [Sov. J. Nucl. Phys. 45, 292 (1987)]; H. David Politzer and Mark. B. Wise, Phys. Lett. B 206, 681 (1988); 208, 504 (1988).

12. Eric Rynes, Senior Honors Thesis, University of Chicago, 1991 (unpublished).

13. C. Quigg and J. L. Rosner, Phys. Reports 56, 167 (1979).

14. A. Martin, Phys. Lett. 93B, 338 (1980).

15. Peter G. O. Freund and Jonathan L. Rosner, Phys. Rev. Lett. 68, 768 (1992).

16. Davison E. Soper and Jonathan L. Rosner, Phys. Rev. D 45, May 1, 1992.

17. Gerard Jungman and Jonathan L. Rosner, Phys. Lett. B 277, 177 (1992).

18. Projects in Classical Electrodynamics (Physics 324), Spring Quarter, 1991, University of Chicago (bound reproduction of reports).

19. Jonathan L. Rosner, Enrico Fermi Institute report EFI 92-07, January, 1992, submitted to Am. J. Phys. 


\section{JIM AMUNDSON}

We have been studying the consequences of treating the strange quark as a heavy quark in the Isgur-Wise heavy quark formalism. We can test the leading order predictions for $D$ meson semileptonic decays using measurements of the Isgur-Wise function from $B$ meson semileptonic decays. The predictions are only roughly in agreement with the data; however, this is what we would expect since we are neglecting terms of order $\bar{\Lambda} / m_{,} \sim 30 \%$. We are now using the $D$ decays to investigate the size of subleading operators in the theory.

\section{GLENN BOYD}

The realization that the Standard Model violates baryon number at high temperature has driven renewed interest in the dynamics of the electroweak phase transition during the early university. My collaborators and I have calculated the electroweak effective potential to fourth order in the $\mathrm{SU}(2)$ coupling $g$. We find significant corrections to lower order calculations when the Higgs mass is over $100 \mathrm{GeV}$. We have also looked at derivative corrections to the effective action.

\section{GERARD JUNGMAN}

The major component of my past year's work has been concerned with radiative corrections to fermion masses in $S O(10)$ grand unified theories. This is envisaged as part of a general program to study extensions of the standard model which allow massive neutrinos and to understand the constraints placed on such models by the measured fermion masses together with the latest precision data for the Weinberg angle and the QCD coupling. The first part of this program was an analysis of a possible weak-scale violation of a 
global lepton number symmetry, together with M. Luty. $S O(10)$ is a characteristic minimal model in which lepton number becomes part of a gauge symmetry. The results of the $S O(10)$ analysis essentially rule out those models which lead to one low energy Higgs doublet, and the two doublet model is constrained significantly.

Together with J. Rosner, I have also studied certain consequences of technicolor-like models which contain a vector resonance analogous to the $\rho$ of $\mathrm{QCD}$. The resulting vector-like four fermion operators can be constrained by their effect in neutral kaon mixing. Also, if one entertains the natural assumption of an introduction of right-handed neutrinos, together with a quark-like ansatz for the lepton mixing, the new vector-like coupling can lead to measurable consequences for lepton number violating processes such as $\mu \rightarrow e \gamma$.

\section{MIHIR WORAH}

M. Worah worked on a model of the quark mixing matrix based on the possibility that quarks are composite objects (have sub-structure). He is currently investigating the possibility that $\mathrm{CP}$ violation is communicated from leptons to quarks via higher-dimension operators.

\section{B. E. MARTINEC, COLLABORATORS, AND STUDENTS}

Two-dimensional solutions to string theory provide a useful laboratory for testing our ideas about strings. Indeed, one might hope that such $2 \mathrm{~d}$ moc are as useful as their particle field theoretic counterparts have been. One of the key features of solvable string models is the possibility of expressing the theory in terms of free variables, typically the eigenvalue/coordinate of a large-N matrix dynamics. E. Witten has proposed a zero ghost-number sector 
of the Liouville BRST cohomology to be the signature in the world-sheet conformal field theory of the eigenvalue variable. This result was a substantial advance in elucidating the relation between the exact matrix dynamics and the more conventional world-sheet conformal field theory approach. The basic idea is that the zero-ghost sector of physical states forms a ring under operator products which parallels the ring of polynomials in the eigenvalue coordinate. D. Kutasov, N. Seiberg and I [1] extended this observation in two directions: first, to the minimal conformal theories coupled to gravity, providing the beginnings of a Liouville-theoretic explanation of the KdV symmetries found in these theories; and second, we showed how the rest of the physical states could be grouped into modules under the action of the ground ring algebra. In particular the 'tachyon' field S-matrix of the $2 \mathrm{~d}$ string is powerfully constrained by demanding the ground ring relations be satisfied by vertex operator correlation functions in the Liouville theory.

One of the key issues in string theory is whether it can ameliorate the singularities of general relativity. Indeed, it had better do so; any physical theory whose solutions develop singularities is incomplete since one does not know how to evolve the equations beyond the point where the singularity forms. String theory claims to be a complete theory, and thus had better not admit singular solutions. However, E. Witten recently proposed a solution to the classical string equations that is a two-dimensional analogue of the Schwarzschild geometry. The $S O(2,1)$ Wess-Zumino-Witten model (the theory of $2 \mathrm{~d}$ current algebra) yields, when the $S O(2)$ subgroup generated by $J_{3}$ is gauged, a $2 \mathrm{~d}$ nonlinear sigma model on a $2 \mathrm{~d}$ target space with the light-cone structure of the Schwarzschild solution in its maximally extended Kruskal coordinatization. Samson Shatashvili and I have succeeded [2] in reducing the problem of quantization of this theory by reducing it to the 
problem of $2 \mathrm{~d}$ gravity coupled to a free scalar field, versions of which have already been solved by matrix techniques. The Liouville-like theory we find has indefinite sign of the cosmological constant. The cosmological constant $|\mu|$ of Liouville theory is related to the mass of the black hole. The connection to Liouville theory could provide the exact solution for correlation functions in the Euclidean black hole geometry using matrix models and Liouville theory; for the Lorentz signature Schwarzschild georsetry it gives us a tool to investigate the properties of singularities in string theory. It is our hope that we can use this exact solution to discover the fate of black hole singularities in string theory. An obstacle to this program is that the Liouville potential we found is in a strongly-coupled regime of the Liouville field theory, which cannot be treated using current techniques.

One problem of this simple model (and indeed a generic feature of all recently investigated 'singular' solutions of string theory) is that the string coupling constant diverges at the core of the singular object. Hence classical physics breaks down and one needs a full quantum field theoretic treatment of the dynamics. One may hope that the collective field theory formulations of two-dimensional string theory might enable us to make progress. No collective field theory has as yet arisen for the black hole solution; however, one may try to produce a candidate based on the information gathered from conformal field theory calculations. This is a rather formidable task for the black hole background, as very little is known to date about the noncompact gauged Wess-Zumino-Witten model. Instead, I set the somewhat simpler task of finding a collective field theory for the $2 \mathrm{~d}$ fermionic string, about which a great deal is kncwn. One possible byproduct of this study might be a collective field theory for $2 \mathrm{~d}$ superstrings. Working in collaboration with M. Douglas, D. Kutasov, and N. Seiberg, a study was undertaken [3] 
of the properties of the supersymmetric Liouville theory. We succeed to understand much of the string tree-level and one-loop structure of the theory, resulting in a collective field theory ansatz remarkably similar to the bosonic $2 \mathrm{~d}$ string collective field theory. This similarity can largely be traced to the identical ground ring structure of the two theories (see above). However, the ansatz has yet to explain the appearauce of several different modular invariants for the fermionic string (depending on how the world-sheet spin structures are correlated with the sums over the string zero modes;, so the work is as yet incomplete.

\section{JADWIGA BIEŃKOWSKA}

During the last academic year I have worked on several problems concerning $\mathrm{N}=2$ SUSY string models. The larger symmetry group makes $\mathrm{N}=2 \mathrm{SUSY}$ theories simpler to work with than less symmetrical ones, and one expects that $N=2$ SUSY string model will be completely solvable and will lead to a better understanding of string theories. A relationship between the world sheet and space-time symmetries seems to be revealed more directly in $\mathrm{N}=2$ SUSY string theory than in less symmetric models. Also $\mathrm{N}=2 \mathrm{SUSY}$ critical strings in $(2,2)$ dimensions are known to provide a consistent quantum theory of self-dual gravity in four dimensions.

One characteristic feature of $N=2$ SUSY critical string theory is the finite number of physical states. The problem can be formulated as a no-ghost theorem for the $\mathrm{N}=2$ SUSY critical strings. The proof for flat Minkowski space is a generalization of the proofs for $N=0,1$ cases, and confirms the belief that the only physical state with non zero momentum is the Kähler potential deformation. I have proved a generalization of the no-ghost theorem for an arbitrary target space geometry using the larger $\mathrm{N}=4$ superconformal 
algebra present in this model.

\section{PETER BOWCOCK}

The problem of modelling tunneling phenomena in more than one dimension was examined in collaboration with Ruth Gregory, a Fermi/McCormick Fellow in the Relativity Group at the University of Chicago. These methods were generaiized to allow for complex momenta, and possible applications were discussed.

The classification of quantum W-algebras was investigated in collaboration with G. M. T. Watts, at the University of Durham.

Reductive W-algebras generated by bosonic fields of spin 1, a single spin2 field, and fermionic fields of spin $3 / 2$ were classified. Three new cases werc found: a "symplectic" iamily of superconformal algebras, an $N=7$ and an $N=8$ superconformal algebra. The exceptional cases can be viewed as arising from a Drinfeld-Sokolov type reduction of the exceptional Lie superalgebras $F(4)$ and $G(3)$, and have an octonionic description. The quantum versions of the superconformal algebras were constructed explicitly in all three cases.

\section{R. SACHS, COLLABORATORS, AND STUDENTS}

1. Origin of the strong CP problem (R.G.S.)

As reported earlier it has been shown that the $P, T$, and $C$ properties of the $\theta$ vacuum are such that $\mathrm{P}$ cannot be a physical operator of conventional QCD if $\theta \neq 0$ since it does not satisfy the superselection rule $\Delta \theta=0$. Thus parity is not an observable of conventional QCD.

PRL referees have continued to raise technical issues concerning my attempt (reported in my previous progress report as reference [1], Enrico Fermi 
Institute report EFI 92-42) to restore the meaning of parity for arbitrary $\theta$. To overcome this difficulty, the paper has been rewritten for PRD giving much more detail and shifting the emphasis, although the physical consequences are the same as reported earlier [1]. In particular, I emphasize a previously unrecognized $U(2)$ symmetry of the Hilbert space produced by the dynamical solutions of QCD and associated with the topology of the gauge fields. The conventional boundary condition (which assumes that the sign of $\theta$ is fixed) breaks this symmetry and thereby causes the $\mathrm{P}$ and $\mathrm{T}$ violation. If a boundary conditicis preserving the topological symmetry is assumed then the $\mathrm{P}$ and $\mathrm{T}$ violation is found to be due to a chiral rotation $\chi$ of the mass term in the Hamiltonian which is independent of $\theta$. The separation of these two parameters greatly eariches the theory and offers an opportunity to measure each of them. Therefore whether the conventional or the symmetry-preserving boundary condition is the correct one can be decided by experiments.

\section{Alternate phenomenological theory of CP violation (R.G.S.)}

A "natural" phenomenological theory involving no complex coupling constants (they are all real numbers) has been constructed by making use of the chiral rotation of the quarks that is "freed" by the $U(2)$ symmetry-preserving boundary condition on QCD [1]. The theory fits into the structure of the Standard Model but requires two independent Higgs fields. As a consequence of the constraint imposed by the experimental upper limit on the electric dipole moment of the neutron, the results of the theory for $K$ meson phenomena are equivalent to those of a superweak theory. The consequences of the theory for $B$ meson phenomena are under investigation and preliminary results indicate that $\mathrm{CP}$ violation effects in this case may be large. A paper 
reporting these results is under preparation and only awaits confirmation of these estimates [2].

3. Renormalization of neutron edm calculations (M. Booth).

Calculation of the magnitude of the electric dipole moment (edm) of the neutron predicted by either the KM model or the Weinberg model must take into account renormalization effects arising from Weinberg operators. The calculation of the effect of the 3 gluon operator that has been carried out for the latter model required the calculation of many diagrams and its extension to the KM model (4 gluon operators) by the same methods would require many more diagrams. Booth has shown how these calculations can be greatly simplified and has completed the calculation of the anomalous dimension for each case [3]. He is now using the results to determine the effect on calculations of the edm for the KM model. [See also the report by Mr. Booth, helow.]

1. R. G. Sachs, The Origin of Strong CP Violation, Enrico Fermi Institute report EFI 92-21 (submitted to Phys. Rev. D1).

2. R. G. Sachs, The Origin of CP Violation? (in preparation).

3. M. Booth, Anomalous Dimensions of Weinberg Operators, Phys. Rev. D 45, 2518 (1992).

\section{MICHAEL BOOTH}

Over the past year I have completed the study of Weinberg operators in the standard model. Most unportantly, I computed the anomalous dimensions of all dimension-eight gluonic and mixed gluonic-photonic $P$ and $T$ 
violating operators. In fact the result trivially extends to cover all dimensioneight purely gauge-boson vertices, for example a photon may be replaced with a $Z^{0}$. Such operators may be called generalized Weinberg operators. One of the purely gluonic operators has a positive anomalous dimension, which means that it is enhanced during the evolution to lower energy scales. This is in contrast to the original dimension-six Weinberg operator, which is suppressed by the evolution. As a consequence, there are some instances where the dimension-eight operators give the dominant contribution to the neutron electric dipole moment (NEDM). This work is summarized in publications Phys. Rev. D45, 2518 and EFI preprint 92-13, "A note on Weinberg Operators in the Standard Model". 


\section{RECENT PUBLICATIONS}

(* denotes an updated reference from last Progress Report)

\section{JADWIGA BIEŃKOWSKA}

The Renormalization Group Flow in 2-d, $N=2$ SUSY Landau-Ginsburg Models, ElFI-90-62-Rev, August, 1991, submitted to Nucl. Phys. B.

The Generalized No Ghost Theorem for $N=2$ SUSY Critical Strings, Enrico Fermi Institute report EFI-91-65, November, 1991, to be published in Phys. Lett. B.

\section{MIKE BOOTH}

Anomalous Dimensions of Weinberg Operators, D 45, 2518 (1992).

A note on Weinberg Operators in the Standard Model, Enrico Fermi Institute report EFI 92-13.

\section{PETER BOWCOCK}

"Multidimensional Tunneling and Complex Momentum, Peter Bowcock and Ruth Gregory, Phys. Rev. D 44, 1774 (1991).

Tunneling from Nonlocalized Initial States, Peter Bowcock and Ruth Gregory, FERMILAB-PUB-91-142-A, April, 1991.

On The Classification of Quantum $W$ Algebras, P. Bowcock and G. M. T. Watts, Enrico Fermi Institute report EFI-91-63, November, 1991.

Exceptional Superconformal Algebras, Enrico Fermi Institute report EFI92-09, February, 1992. 


\section{GLENN 3OYD}

Aspects of Heavy Quark Physics, Ph. D. Thesis, Caltech, 1991.

$\Omega_{B}$ Semileptonic Decay Form-Factors for $m_{c} \neq \infty$, C. Glenn Boyd and David E. Brahm, Phys. Lett. B254, 468 (1991).

Vanishing of $1 / \mathrm{m}$ Corrections at Threshold, C. Glenn Boyd and David E. Brahm, Phys. Lett. B257, 393 (1991).

Infrared Divergences in the Electroweak Phase Transition, C. G. Boyd, D. E. Brahm, and S. D. Hsu, EFI 92-22, submitted to Nucl. Phys. B.

\section{GEOFFREY HARRIS}

*Resolving a Two-fold Ambiguity in CP Violation Parameters, with Jonathan L. Rosner, Phys. Rev. D 45, 946 (1992).

\section{GERARD JUNGMAN}

Nonexistence Theorems for Asymptotically Euclidean Einstein-Matter Solutions (with R. Wald), Phys. Rev. D 40, 2615 (1989).

Massive Neutrinos and the Weak Scale Singlet Majoron, with M. A. Luty, Nucl. Phys. B361, 24 (1991).

*New Vector-Like Interactions: Constraints from Kaon Physics and from $\mu \rightarrow$ eq with Jonathan L. Rosner, Phys. Lett. B277, 177 (1992).

Further Topological Proofs of Gribov Ambiguities, Mod. Phys. Lett. A7, 849 (1992).

Fermion Masses in $S O(10)$, Enrico Fernii Institute report EFI 91-64 submitted to Phys. Rev. D.

Fermion Masses in $S O(10)$ : The Case of the Tree Level Relation $m_{b}=3 m_{\tau}$, Enrico Fermi Institute report EFI 92-18, in preparation. 


\section{PYUNGWON KO}

" $K_{L} \rightarrow \mu^{+} \mu^{-}$, the top-quark mass, and other related topics, Phys. Rev. D 45, 1174 (1992).

\section{EMIL MARTINEC}

"Black Hole Physics and Liouville Theory, with Samson L. Shatashvili, Nucl. Phys. B368, 338 (1992).

An Introduction to 2-d Gravity and Solvable String Models, Rutgers University report RU-91-51, September, 1991, Lectures at the 1991 Trieste Spring School on String Theory and Quantum Geometry, Trieste, Italy, Apr 15-23, 1991.

Ground Rings and their Modules in 2-d Gravity with $c \leq 1$ Matter, with David Kutasov and Nathan Seiberg, Phys. Lett. B276, 437 (1992).

A Noncritical Fermionic String Ansatz, with M. Douglas, D. Kutasov, and N. Seiberg, work in progress.

\section{ROGERIO ROSENFELD}

*Adler-Weisberger Relation for Scattering of Longitudinal $W$ Bosons, with Jonathan L. Rosner, Phys. Rev. D 44, 2133 (1991).

*Technirho Production at SSC and LHC, with Jonathan Bagger and T. Han, in Research Directions for the Decade (Proceedings of the 1990 DPF Snowmass Workshop), edited by E. L. Berger.

\section{JONATHAN L. ROSNER}

"Impact of New $\left|V_{u b} / V_{c b}\right|$ and $\epsilon^{\prime} / \epsilon$ Measurements on Weak Mixing Angles, with C. S. Kim and C.-P. Yuan, Phys. Rev. D 42, 96 (1990); 45, 389(E) (1992). 
"Particle Physics at the Crossroads, in Trends in Theoretical Physics, vol. 2, Ed. by P. J. Ellis and Y. C. Tang (Addison-Wesley, Redwood City, CA, 1991), p. 141.

*Atomic Parity Violation as a Probe of New Physics, with William J. Marciano, Phys. Rev. Lett. 65, 2963 (1990); 68, 898(E) (1992).

*Tests of a Composite Model of Quarks and Leptons, with Davison E. Soper, Phys. Rev. D 45, May 1, 1992, to be published.

*Probing New Physics with Precise Electroweak Tests, in Intersections between Particle and Nuclear Physics, Tucson, AZ, May 24-29, 1991, edited by W. T. H. van Oers (AIP, New York, 1992), p. 156.

*The Densities of Meson and Baryon States, with Peter G. O. Freund, Phys. Rev. Lett. 68, 765 (1992).

*The Cabibbo-Kobayashi-Maskawa Matrix, in B Decays, ed. by Sheldon L. Stone (World Scientific, Singapore, 1992).

The CKM Matrix and $B$ Physics, Enrico Fermi Institute report EFI-92-02, January, 1992, invited talk at Workshop on $b$ Physics, Edinburgh, Scotland, Dec. 9, 1991, to be published in J. Phys. G.

Ratios of Observables for $B$ Mesons, with P. F. Harrison, Enrico Fermi Institute report EFI-92-05, February, 1992, to be published in J. Phys. G.

The Smith Chart and Quantum Mechanics, Enrico Fermi Institute report EFI-92-07, January, 1992, submitted to Am. J. Phys.

Models of the Quark Mixing Matrix, with Mihir Worah, Enrico Fermi Institute report EFI-92-12, March, 1992, submitted to Phys. Rev. D.

Electroweak Measurements and the Top Quark, Enrico Fermi Institute report EFI 92-19, April, 1992, to be submitted to Rev. Mod. Phys. (Colloquia). 
See also: Geoffrey Harris, Gerard Jungman, Rogerio Rosenfeld

\section{ROBERT G. SACHS}

The Origin of Strong CP Violation, Enrico Fermi Institute report EFI 92-21 (submitted to Phys. Rev. D).

The Origin of CP Violation? (in preparation).

\section{ANNE TAORMINA}

"Modular Invariant Partition Functions for the Doubly Extended $N=4$ Superconformal Algebras, with Hirosi Ooguri and Jens Lyng Petersen, Nucl. Phys. B368, 611 (1992). 

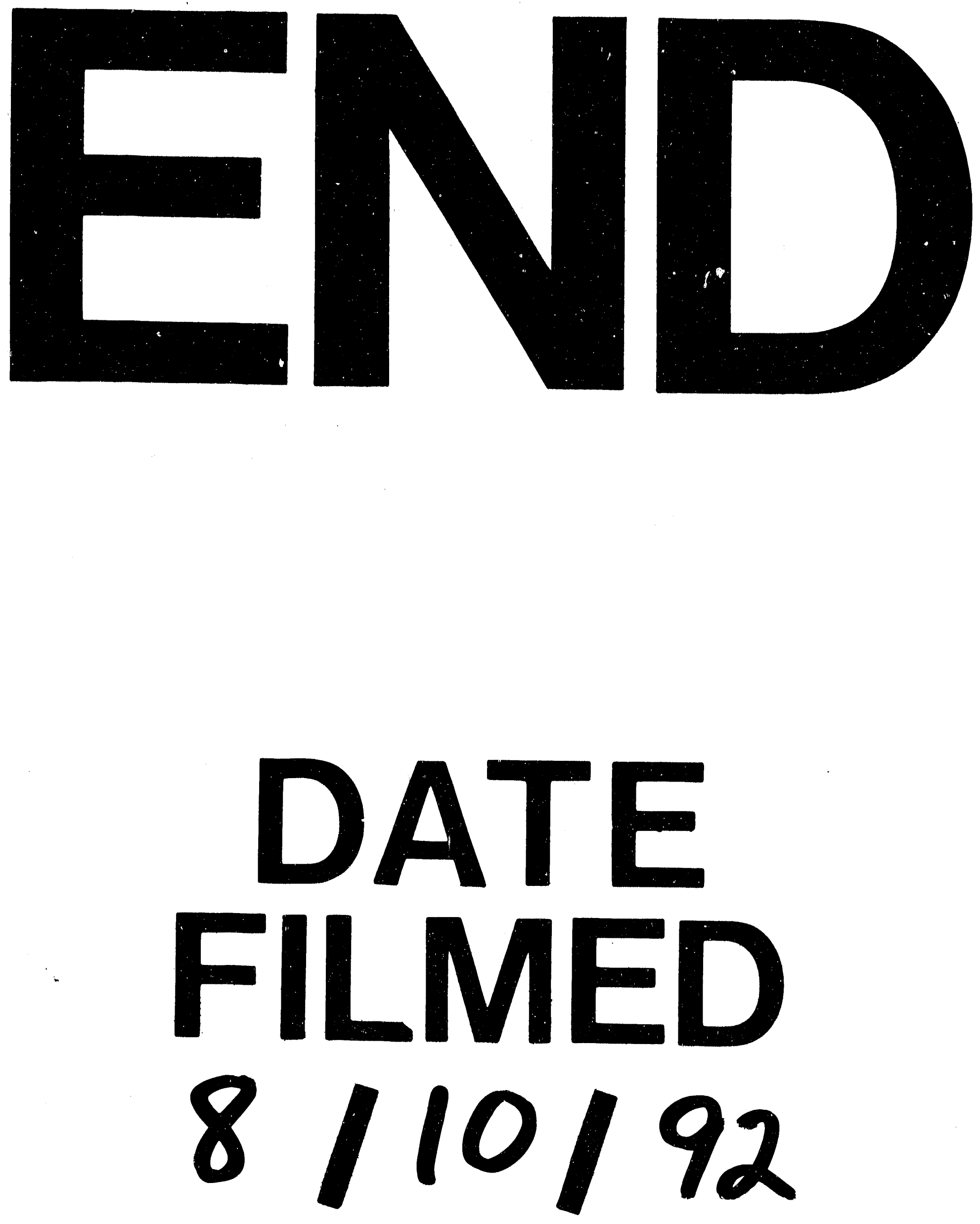
